\title{
Theory of nucleation and growth during phase separation
}

\author{
Celeste Sagui and Martin Grant \\ Centre for the Physics of Materials, Physics Department, Rutherford Building, McGill University, 3600 rue University, \\ Montréal, Québec, Canada H3A 2T8
}

(Received 26 May 1998)

\begin{abstract}
We present a new model for the entire process of phase-separation that combines steady-state homogeneous nucleation theory with the classical Lifshitz-Slyozov mechanism of ripening, modified to account for the substantial correlations among the droplets. A set of self-consistent interface equations describes the decay of metastable states, incorporating naturally the crossover from early-stage nucleation to the late-stage scaling regime without ad hoc assumptions. We present simulation results for both two and three dimensions. We also present a mean-field, Thomas-Fermi approximation that provides an approximate solution to the many-body problem. [S1063-651X(99)05503-8]

PACS number(s): 64.60.My
\end{abstract}

\section{INTRODUCTION}

The theory of homogeneous nucleation has been a subject of research for at least 60 years [1]. A metastable state evolves towards the stable equilibrium state via localized droplet fluctuations of a critical size. The critical energy for the formation of a droplet is determined by a competition between a volume term (which favors creation of the droplet) and a surface term (which favors its dissolution). The critical radius $R_{c}$ results from this competition: droplets of size $R$ $>R_{c}$ grow, while droplets with $R<R_{c}$ shrink. Early theories of homogeneous nucleation [1] have been generalized and made rigorous, particularly through the work of Langer [2]. Experimental tests [3] and computer simulations [4] are well in accord with predictions. At present, the process of earlytime homogeneous nucleation is quite well understood.

This is also the case for late-time phase separation, known as Ostwald ripening. During this late stage, droplets coarsen while maintaining local equilibrium. To reduce the system interfacial free energy, material diffuses away from small high-curvature droplets, which shrink and dissolve. This material condenses onto large low-curvature droplets, which grow. This mechanism was first described by Lifshitz and Slyozov, and Wagner [5], in the limit of volume fraction $\phi$ $\rightarrow 0$, where interactions between droplets through their diffusion fields can be neglected. Since then, these interactions have attracted much research. The main results [6-8] are that the universal scaling form of the droplet distribution function predicted by Lifshitz and Slyozov depends on $\phi$, and that the predicted $n=1 / 3$ power-law growth for the mean radius has the following form: $R(t)=[K(\phi) t]^{1 / 3}$, for late times, where the coarsening rate $K(\phi)$ is a monotonically increasing function of $\phi$.

Experimentally, nucleation, growth and coarsening have been studied in traditional systems like binary fluids [3], vapor condensation [9], melt crystallization [10], as well as precipitation reactions in supersaturated alloys [11]. Nontraditional applications of these ideas have been made in the study of glasses and amorphous materials [12], in cavities in metastable viscous fluids with modulated pressure [13], and in three-dimensional clusters on two-dimensional surfaces [14].
With these two regimes of early and late time being reasonably well understood, the question remains: How does the system proceed from nucleation to the late stage of Ostwald ripening? This was first addressed in the seminal work of Langer and Schwartz [15], who used a mean-field approach to study the nonlinear dynamical equations of motion for a phase separating system with both nucleation and growth of droplets. There are more recent models and experiments concerning the description of nucleation and growth within a single framework. For instance, chemical reaction rate theory has been used, within a mean-field framework, to model the kinetics of precipitation reactions in Al alloys [16]. Many mean-field theories [17] are modifications of the formalism first proposed by Kolmogorov, Johnson and Mehl, and Avrami [1]. They involve isothermal, time transformation relations that relate the volume fraction of the transformed phase at a given time with growth rate, nucleation frequency, and shape factors. Such theories consider systems where diffusive effects within the matrix, from either monomer diffusion or the release of latent heat from droplets, can be neglected. That is, unlike the present situation, any Ostwald ripening regime is of negligibly short duration.

None of these mean-field theories includes correlations between droplets. Experimental evidence [18], however, emphasizes the importance of interparticle diffusional interactions and of particle spatial locations on nucleation and growth, and thus, the need for a theory to include such correlation effects. Tokuyama and Enomoto [19] studied the effects of correlations on the kinetics of the crossover regime for a three-dimensional system. However, their study did not include nucleation and was based on a perturbative expansion in the volume fraction $\phi$, to order $\sqrt{\phi}$.

In this paper [20], we introduce a new model that combines steady-state homogeneous nucleation theory with the classical Lifshitz-Slyozov mechanism, modified to account for the substantial correlations amongst the droplets. Our model is formulated in terms of a set of self-consistent interface equations, which are then solved numerically both in two dimensions (2D) and in three dimensions (3D). This new formalism naturally incorporates the crossover from the early-stage nucleation regime to the late-stage scaling regime. We also present a mean-field, Thomas-Fermi approxi- 
mation that includes these correlation effects. We note that we have not incorporated elastic effects, which are important in some instances. It is straightforward to generalize our approach to include such effects, if they are important. This paper is organized as follows. In Sec. II, we introduce our model, while in Sec. III, we describe the simulation method. Our mean-field theory is presented in Sec. IV, while Sec. V gives our results, and concludes the paper.

\section{MODEL}

The energy for the formation of a droplet of radius $R$ has a surface and a volume term:

$$
E(R)=a \sigma R^{d-1}-\frac{v \Delta \mu}{v_{m}} R^{d},
$$

in $d$ dimensions. Herein, $\sigma$ is the surface tension, $v_{m}$ is the molecular volume, $v=\pi^{d / 2} / \Gamma(d / 2+1), \quad a=d v, \Delta \mu$ $\simeq k T\left(C(t)-C_{e q}(\infty)\right) / C_{e q}(\infty)$ is the variation in chemical potential, $k$ is Boltzmann's constant, $T$ is the temperature, $C(t)$ is the time-dependent supersaturation, and $C_{e q}(\infty)$ is the solute concentration in the matrix at a planar interface in a phase-separated system. The energy has a maximum at a critical radius $R_{c}$ given by

$$
R_{c}=\frac{(d-1) v_{m} \sigma}{\Delta \mu}
$$

such that the critical energy $E\left(R_{c}\right) \equiv E_{c}=v \sigma R_{c}^{d-1}$.

Our study makes use of dimensionless variables. Units of length and time are given in terms of the capillary length $l_{c}=(d-1) \sigma v_{m} /(k T)$ and the characteristic time $t_{c}$ $=l_{c}^{2} /\left[D C_{e q}(\infty) v_{m}\right]$, where $D$ is the diffusion coefficient. We also introduce a dimensionless concentration field $\theta(\mathbf{r}, t)$ $=\left[C(\mathbf{r}, t)-C_{e q}(\infty)\right] / C_{e q}(\infty)$, whose value far from any droplet is the time-dependent supersaturation $\chi(t)$, and the dimensionless parameter $\chi_{0}^{d-1}=v \sigma l_{c}^{d-1} /(k T)$. Expressed in dimensionless form (i.e., $R_{c} / l_{c} \rightarrow R_{c}$ ), the critical radius becomes

$$
R_{c}=\frac{1}{\chi(t)}
$$

and the dimensionless energy ratio $E_{c} / k T$ can be expressed as

$$
\frac{E_{c}}{k T}=\left(\frac{\chi_{0}}{\chi(t)}\right)^{d-1}
$$

The relation between $\chi(t), \chi_{0}$ and the corresponding parameters used by Langer and Schwartz, $\chi^{*}(t), \chi_{0}^{*}[15]$, is $\chi(t)$ $\simeq(\beta / 2) \chi^{*}(t)$ and $\chi_{0} \simeq(\beta / 2) \chi_{0}^{*}$, where $\beta$ is the critical exponent for temperature dependence of the concentration near the critical temperature $\left(\Delta C \sim\left|T-T_{c}\right|^{\beta}\right)$. For 3D, Langer and Schwartz used $\beta=1 / 3$ and $\chi_{0}^{*} \sim 1$ which gives $\chi_{0} \sim 1 / 6$ for our parameter in $3 \mathrm{D}$. Note that $\chi_{0}^{d-1}$ determines the intensity of noise, since it is proportional to $1 / T$.

The nucleation rate gives the number of droplets nucleated per unit volume per unit time for a given supersaturation. It has the general form

$$
J=\frac{1}{\tau} \Omega e^{-\left(E_{c} / k T\right)}
$$

where $\tau$ is the time scale for the macroscopic fluctuations, $\Omega$ is the volume of phase space accessible for fluctuations, and $e^{-\left(E_{c} / k T\right)}$ is the Boltzmann probability factor for nucleation of a droplet. The field-theoretic steady-state nucleation rate has been studied extensively in the literature $[2,15,21]$. Here we reproduce the results in dimensionless form for space dimension $d$ :

$$
J_{d}(t)=A_{d}\left(\frac{\chi(t)}{\chi_{0}}\right)^{\alpha_{d}} \beta_{d} \exp \left[-\left(\frac{\chi_{0}}{\chi(t)}\right)^{d-1}\right]
$$

where $\alpha_{3}=2 / 3, \alpha_{2}=4, \beta_{3}=\left(1+\chi(t) / \chi_{0}\right)^{3.55}, \beta_{2}=1$, and $A_{d}=\chi_{0}^{d+3} / v$ is a constant. The nucleation rate $J_{d}(t)$ can be written as a radial integration of a distributed nucleation rate $j_{d}(R, t)$ :

$$
J_{d}(t)=\int_{0}^{\infty} j_{d}(R, t) d R
$$

A reasonable assumption for $j_{d}(R, t)$ is a Gaussian form, i.e.,

$$
j_{d}(R, t)=\frac{1}{\sqrt{2 \pi}(\delta R)} \exp \left[-\frac{\left(R-R_{c}\right)^{2}}{2(\delta R)^{2}}\right] J_{d}(t)
$$

Considered only as a function of radius, the droplet energy (1) is a maximum at the critical radius, and thus $(\delta R)^{2}$ can be written as $(\delta R)^{2}=2\left[E_{c}-E(R)\right] /\left|E_{c}^{\prime \prime}\right|$, where $E_{c}$ and $E_{c}^{\prime \prime}$ are the functions $E(R)$ and its second derivative evaluated in $R=R_{c}$. Langer [2] showed that when the droplet energy is not only a function of radius $R$ but also of capillary wavelength fluctuations $w$, then droplets appear at the saddle point in the surface $E(R, w)$. In this case, the surface of the droplet is described by a function $a\left[R \sqrt{1+(w / R)^{2}}\right]^{d-1}$ so that the change in the droplet energy due to nonzero $w$ is $\Delta E(R)$ $=a \sigma R^{d-1}(d-1)(w / R)^{2} / 2$. Both approaches lead to the same width of the distribution (with $w \equiv \delta R$ ), that can be computed as that corresponding to an uncertainty in the activation energy of the order of $k T / 2$. In dimensionless form, it is

$$
(\delta R)^{2}=\frac{R_{c}^{3-d}}{d(d-1) \chi_{0}^{d-1}}
$$

In our study, we consider different forms of $\delta R$, since different initial distributions of droplets can lead to very different intermediate regimes, and it is possible to envision many different experimental situations in which the width of the distribution does not necessarily follow Eq. (9). For example, it is possible to adjust the polydispersivity of the distribution by quenching in prescribed steps in temperature, and by incorporating some degree of heterogeneous nucleation.

Next, we consider the growth and ripening problem. In order to generalize the Langer-Schwartz theory to nonzero volume fraction, one needs to determine the diffusional interaction of a droplet with its surroundings. The time evolution of the system is described by the multi-droplet diffusion 
equation for the concentration field $\theta(\mathbf{r}, t)$. In the monopole approximation, the coarsening phase is spherical and fixed in space. The emission or absorption of solute from growing or dissolving particles is modeled by placing point sources or sinks of solute at the center $\mathbf{r}_{i}$ of each particle $i$. The multidroplet diffusion equation then becomes

$$
\frac{\partial \theta(\mathbf{r}, t)}{\partial t}-\nabla^{2} \theta(\mathbf{r}, t)=-a \sum_{i=1}^{N(t)} Q_{i} \delta\left(\mathbf{r}-\mathbf{r}_{i}\right),
$$

where the coefficients $Q_{i}$ describe the strength of the source or sink of the current for diffusion. We assume spherical droplets in local equilibrium; hence, the concentration near the interface is determined by the local curvature and surface tension, consistent with the Gibbs-Thompson boundary condition:

$$
\theta\left(\mathbf{R}_{i}\right)=1 / R_{i}
$$

in dimensionless form.

The radial growth law is obtained from a local continuity equation in a volume that encloses only one droplet:

$$
R_{i}^{d-1} \frac{d R_{i}}{d t}=Q_{i}
$$

If $\phi$ is the constant volume fraction of the minority phase, the conservation of mass requires

$$
\chi(t)+v \sum_{i=1}^{N(t)} R_{i}^{d}=\phi
$$

The time derivative of the second term on the left-hand side gives two contributions: one due to the growth or dissolution of existing droplets, $a \sum_{i=1}^{N(t)} R_{i}^{d-1} \dot{R}_{i}$, and another due to the time variation of the number of droplets $N(t)$ during nucleation, which we shall call $a Q_{n u c l}$, and whose expression is given later in Eq. (23). Thus, the time derivative of Eq. (13) is

$$
\frac{\partial \chi(t)}{\partial t}+a \sum_{i=1}^{N(t)} Q_{i}+a Q_{n u c l}=0
$$

the supersaturation $\chi(t)$ varies due to either nucleation of new droplets via $\partial \chi /\left.\partial t\right|_{n}=-a Q_{n u c l}$, or growth/dissolution of existing droplets via $\partial \chi /\left.\partial t\right|_{g}=-a \sum_{i=1}^{N(t)} Q_{i}$.

Equations (6), (10), (11), (12), and (14) contain all the elements necessary to describe the phase separation of the system from the initial nucleation regime to the late-time Ostwald ripening regime. First, we need to provide a solution for Eq. (10). In the Appendix, we give a formal solution for this equation, Eq. (A2), that employs a retarded Green's function. When this solution is averaged over the surface of the $i$ th particle, one obtains Eq. (A5), which we rewrite here for the three-dimensional case:

$$
\frac{Q_{i}}{R_{i}}=\chi(t)-\frac{1}{R_{i}}-4 \pi \sum_{j \neq i}^{N} \int_{0}^{t} d s \exp \left(-\frac{\left|\mathbf{r}_{i}-\mathbf{r}_{j}\right|^{2}}{4 s}\right) \frac{Q_{j}(t-s)}{(4 \pi s)^{3 / 2}}
$$

In this equation an approximation has been made: the timedependent Green's function is used in the integration of all the particles $j$ with $j \neq i$, while in particle $i$ it is replaced by the corresponding stationary Green's function. This equation, together with Eq. (12), was used in Ref. [19] to study the evolution of a three-dimensional system with a given, initial distribution of particles. It represents the many-body effects due to the diffusive long-range interactions among droplets, and therefore a closed-form solution cannot be written. The authors employed a systematic expansion in powers of $\sqrt{\phi}$ and solved the equation to first order. Later, we will seek a solution of a similar equation by introducing a Thomas-Fermi approximation.

Here we propose an ansatz for the solution of Eq. (10). Instead of using retarded Green's functions for the solution, we use time-independent Green's functions, specifically $G_{3}\left(\mathbf{r}-\mathbf{r}^{\prime}\right)=1 /\left|\mathbf{r}-\mathbf{r}^{\prime}\right|$ in $3 \mathrm{D}$ and $G_{2}\left(\mathbf{r}-\mathbf{r}^{\prime}\right)=\ln \left(\left|\mathbf{r}-\mathbf{r}^{\prime}\right| / L\right)$ in $2 \mathrm{D}$, where $L$ is the system size. To take into account the time evolution of the supersaturation $\chi(t)$, we introduce a coefficient $Q_{0}(t)$, that will be related to the coefficients $Q_{i}$ through the conservation law. In $3 \mathrm{D}$, the proposed solution reads

$$
\frac{1}{R_{i}}=Q_{0}(t)-\frac{Q_{i}}{R_{i}}-\sum_{j \neq i}^{N(t)} \frac{Q_{j}}{\left|\mathbf{r}_{i}-\mathbf{r}_{j}\right|}
$$

In $2 \mathrm{D}$ it is

$$
\frac{1}{R_{i}}=Q_{0}(t)-Q_{i} \ln \left(\frac{R_{i}}{L}\right)-\sum_{j \neq i}^{N(t)} Q_{j} \ln \left(\frac{\left|\mathbf{r}_{i}-\mathbf{r}_{j}\right|}{L}\right) .
$$

The introduction of the coefficient $Q_{0}(t)$, instead of the retarded Green's function, is based on the assumption that the behavior of the supersaturation field outside the droplets can be described in a mean-field manner. Notice that in the steady-state Ostwald ripening regime $Q_{0}(t) \equiv \chi(t)$, so $Q_{0}(t)$ acts like an "effective" supersaturation, whose variation in time allows for the diffusive growth of particles. Since at late times, $\chi(t) \rightarrow 1 /\langle R(t)\rangle$, where $\langle R(t)\rangle$ is the mean radius, then $C_{0}(t)=Q_{0}(t)-1 /\langle R\rangle \rightarrow 0$ at late times. This quantity is plotted for a particular set of parameters in Fig. 3.

We have numerically tested that there is no measurable difference between the exact solution of Eq. (15) and our ansatz. Hence we used the approximate solution: It is easier to solve numerically. In particular, we note that this ansatz does not force long-range interactions to be present. For the initial stages of nucleation, the $Q_{i}(t)$, through which longrange interactions potentially enter, are practically zero. As time increases, these $Q_{i}(t)$ increase as well, and the system crosses over naturally to a regime where long-range interactions become important.

A mean-field treatment of the variation of the supersaturation means that the variation of $\chi(t)$ due to nucleation, $\partial \chi /\left.\partial t\right|_{n}$, is computed using the theoretical nucleation rate in Eq. (6) according to a simple scheme that we describe in Sec. III, while the variation due to growth or dissolution of existing droplets is given by

$$
\left.\frac{\partial \chi}{\partial t}\right|_{g}=-a \sum_{i=1}^{N(t)} \frac{R_{i}^{d-2}}{C_{d}\left(R_{i}\right)}\left(\chi(t)-\frac{1}{R_{i}}\right)
$$


where $C_{3}\left(R_{i}\right)=1$ in $3 \mathrm{D}$, and $C_{2}\left(R_{i}\right)=\ln \left(L / R_{i}\right)$ in $2 \mathrm{D}$. Defining the average of a function $f(R)$ as $\langle f(R)\rangle$ $=(1 / N) \sum_{i} f\left(R_{i}\right) \quad$ and $\quad\langle\langle 1 / R\rangle\rangle=\left\langle[R \ln (R / L)]^{-1}\right\rangle /$ $\left\langle[\ln (R / L)]^{-1}\right\rangle$, the above equation in $3 \mathrm{D}$ becomes:

$$
\sum_{i=1}^{N(t)} Q_{i}=N\langle R\rangle\left(\chi(t)-\frac{1}{\langle R\rangle}\right) .
$$

In $2 \mathrm{D}$, it becomes

$$
\sum_{i=1}^{N(t)} Q_{i}=\left\langle[\ln (L / R)]^{-1}\right\rangle N[\chi(t)-\langle\langle 1 / R\rangle\rangle] .
$$

Equations (16) and (19) in 3D, and (17) and (20) in 2D, represent a set of $N+1$ linear coupled equations for the $N$ coefficients $Q_{i}$ and the coefficient $Q_{0}$. Together with the growth equation Eq. (12) and the nucleation rate equation Eq. (6), they constitute a formal solution to the nucleation and growth problem.

The multiparticle diffusion problem can be solved using a multipole expansion method valid to an arbitrary order of the expansion. We shall only consider the lowest-order term in this expansion, the monopole approximation, which is reasonable for volume fractions $\phi \leqslant 0.10$, as shown in the study of Akaiwa and Voorhees [8]. Higher-order approximations, useful for the study of larger volume fractions, can be handled following their approach. Qualitatively, the multipole expansion behaves as follows. To the next order, a dipole term appears which leads to a nonuniform concentration gradient along the surface of the (still spherical) droplets, which now migrate with a velocity linearly dependent on coefficients from first-order spherical harmonics [8]. Higherorder multipole terms result in nonspherical droplets.

\section{SIMULATIONS}

In the previous section, we obtained a set of equations for the description of nucleation, diffusive growth and ripening in a phase-separating system. The numerical solution of similar sets of self-consistent equations for the Ostwald ripening regime has been described in detail in the literature $[7,22]$, so here we shall describe only the main differences with the previous simulation procedures.

As in previous work, the simulation results do not depend on the system size $L$, provided length and time in the system are rescaled by $L$ and $L^{3}$, respectively, implying that the nucleation rate in Eq. (6) is rescaled by $1 / L^{d+3}$. When choosing the edge length of the box enclosing the system, certain care must be taken, however. The dimensions of the vectors and matrices in the code depend on the maximum number $N_{\max }$ of droplets that can be nucleated. Therefore, one chooses $N_{\max }$ according to computational limitations, and chooses the edge length $L$ that can accommodate that number for a given volume fraction $\phi$. Hence, $L$ must be optimized with trial runs indicating the number of droplets $N(t)$ nucleated, and their maximum number. With a convenient $L$, relevant quantities are properly rescaled as, for example, $J_{d}(t) \rightarrow J_{d}(t) L^{d+3}, R_{c}(t=0) \rightarrow 1 /(\phi L)$, and production runs can be done.

For homogeneous nucleation, the time evolution starts in an initially supersaturated state, $\chi(t=0)=\phi$. The equations are integrated numerically using an Euler discretization scheme with a variable time increment $d t$. That increment is the smaller of two time intervals: the time needed to nucleate $d t_{n}$, or eliminate $d t_{r}$, one droplet. Since the nucleation rate represents the number of nucleated droplets per unit volume per unit time at a given supersaturation $\chi(t)$, we have

$$
d t_{n}=\frac{1}{J_{d}[\chi(t)]}
$$

Note that the scaled volume of the system is unity. The integration of the growth equation for droplet $i$ gives $R_{i}^{d}(t$ $+d t)=R_{i}^{d}(t)+\left(Q_{i} d\right) d t$. Hence,

$$
d t_{r}=\min \left\{-\frac{R_{i}^{d}}{Q_{i} d}\right\}
$$

for all $i$. This will typically be the smallest droplet, which will usually have the largest negative current for growth $Q$, along with the smallest $R$. Occasionally, however, the proximity of nearby droplets may lead to a particularly large $Q$ for a larger droplet, implying a different $d t_{r}$.

The actual time interval $d t$ is the smaller of $d t_{n}$ and $d t_{r}$. Suppose the minimum time interval favors nucleation of a droplet. Then the radius of the droplet $R_{\text {drop }}$ is chosen with the Box-Muller method for generating random deviates with a Gaussian distribution consistent with Eq. (8). Each coordinate is chosen as a uniform random deviate between 0.0 and 1.0, avoiding overlap with existing droplets [23]. The nucleated droplet represents a small fraction of the supersaturation, whose new value is $\chi\left(t+d t^{*}\right)=\chi(t)-v R_{d r o p}^{d}$. Thus, we identify $Q_{\text {nucl }}$ in Eq. (14) as

$$
Q_{n u c l}=\frac{R_{d r o p}^{d}}{d d t^{*}}
$$

Radii $R_{i}$ and supersaturation $\chi(t)$ are updated following the appropriate $d t$. The minimum $d t$ must be computed selfconsistently. For instance, immediately after a nucleation process, or the integration of the radial growth equation, one must compute a new set of coefficients $\left\{Q_{i}\right\}$ that, naturally, modifies the time interval $d t_{r}$. One must also compute a new time interval $d t_{n}$, since the supersaturation has changed. This "time bookkeeping" permits the self-consistent selection of the interval $d t$ : the path of minimum $d t$ is completely determined by the dynamical equations of the system.

\section{MEAN-FIELD APPROXIMATION}

In this section we extend a mean-field approach used for the late time Ostwald ripening regime [7] to include the nucleation and crossover regimes. For nonzero volume fraction $\phi$, droplet-screening and many-droplet correlation effects are approximated in the same manner as the ThomasFermi mechanism for Coulomb systems. Within a mean-field approximation, the change in volume of a droplet depends on the concentration gradients set up by all the droplets, that can be written as $\sum_{j} I_{\text {int }}\left(R_{i}, R_{j}\right)\left[\chi(t)-1 / R_{j}\right]$, where $I_{\text {int }}\left(R_{i}, R_{j}\right)$ is the interaction matrix. In the mean-field approximation, all 
droplets are equivalent, so $I_{\text {int }}\left(R, R^{\prime}\right) \propto I(R) \delta_{R, R^{\prime}}$. The growth law then becomes

$$
\frac{d\left(v R_{i}^{d}\right)}{d t}=I\left(R_{i}\right)\left(\chi(t)-\frac{1}{R_{i}}\right)
$$

In this approximation, the flux determining the growth rate for each droplet is only proportional to the difference between the boundary concentration and the supersaturation. The rate coefficient $I(R)$ must be determined selfconsistently in terms of the screening length defined below.

To complete the mean-field description, we introduce two continuity equations. One is the continuity equation for the droplet distribution function $f(R, t)$ :

$$
\frac{\partial f(R, t)}{\partial t}+\frac{\partial[\dot{R} f(R, t)]}{\partial R}=j_{d}(R, t)
$$

where $j_{d}(R, t)$ is given by Eq. (8), and the total number of droplets per scaled volume is related to $f(R, t)$ through:

$$
N(t)=\int_{0}^{\infty} f(R, t) d R
$$

The time derivative of the mass conservation equation,

$$
\chi(t)+\int_{0}^{\infty} v R^{d} f(R, t) d R=\phi
$$

gives rise to the other continuity equation:

$$
\frac{\partial \chi(t)}{\partial t}=-a \int_{0}^{\infty} R^{d-1} \dot{R} f(R, t) d R-\int_{0}^{\infty} v R^{d} j_{d}(R, t) d R .
$$

By substituting Eq. (24) above, we get

$$
\begin{aligned}
\frac{\partial \chi(t)}{\partial t}= & -\chi(t) \int_{0}^{\infty} I(R) f(R, t) d R \\
& +\int_{0}^{\infty} I(R) R^{-1} f(R, t) d R-\int_{0}^{\infty} v R^{d} j_{d}(R, t) d R .
\end{aligned}
$$

In the same manner as in Ref. [7], an equation of motion for the local concentration field $\theta(\mathbf{r}, t)$ in the vicinity of the $i$ th droplet is postulated:

$$
\frac{\partial \theta(\mathbf{r}, t)}{\partial t}=\nabla^{2} \theta(\mathbf{r}, t)-\xi^{-2} \theta(\mathbf{r}, t)+S_{b}-S_{n}-a Q_{i} \delta\left(\mathbf{r}-\mathbf{r}_{i}\right),
$$

where the contribution from the other droplets is introduced by a screening length $\xi$, a background field $S_{b} \xi^{2}$, and a source field $S_{n} \xi^{2}$. If we consider the limit of this equation far from any droplet, we obtain

$$
\frac{\partial \chi(t)}{\partial t}=-\xi^{-2} \chi(t)+S_{b}-S_{n},
$$

which, by comparison with Eq. (29), immediately gives

$$
\begin{gathered}
\xi^{-2}=\int_{0}^{\infty} I(R) f(R, t) d R, \\
S_{b}=\int_{0}^{\infty} I(R) R^{-1} f(R, t) d R,
\end{gathered}
$$

and,

$$
S_{n}=\int_{0}^{\infty} v R^{d} j_{d}(R, t) d R
$$

By substituting $S_{b}-S_{n}$ in Eq. (30) and defining the field $\psi(\mathbf{r}, t)=\theta(\mathbf{r}, t)-\chi(t)$, we can rewrite Eq. (30) as

$$
\frac{\partial \psi(\mathbf{r}, t)}{\partial t}=\nabla^{2} \psi(\mathbf{r}, t)-\xi^{-2} \psi(\mathbf{r}, t)-a Q_{i} \delta\left(\mathbf{r}-\mathbf{r}_{i}\right)
$$

The solution of this equation can be expressed in terms of a time-dependent, screened Green's function:

$$
\begin{aligned}
\psi(\mathbf{r}, t)= & -\frac{a}{(2 \pi)^{d}} \int_{0}^{t} d s \int d^{d} k e^{-\left(k^{2}+\xi^{-2}\right) s} \\
& \times e^{i \mathbf{k} \cdot\left(\mathbf{r}-\mathbf{r}_{i}\right)} Q_{i}(t-s),
\end{aligned}
$$

and, making $Q_{i}(t-s) \simeq Q_{i}(t)$,

$$
\psi(\mathbf{r}, t)=-Q_{i} V\left(\mathbf{r}-\mathbf{r}_{i}, \xi, t\right),
$$

where

$$
V\left(\mathbf{r}-\mathbf{r}_{i}, \xi, t\right)=-\frac{a}{(2 \pi)^{d}} \int d^{d} k \frac{1-e^{-\left(k^{2}+\xi^{-2}\right) t}}{\left(k^{2}+\xi^{-2}\right)} e^{i \mathbf{k} \cdot\left(\mathbf{r}-\mathbf{r}_{i}\right)} .
$$

For $t \gg 1, V\left(\mathbf{r}-\mathbf{r}_{i}, \xi, t\right)$ can be identified with the steadystate, screened Green's function, $V\left(\mathbf{r}-\mathbf{r}_{i}, \xi\right)=\exp (-\mid \mathbf{r}$ $\left.-\mathbf{r}_{i} \mid / \xi\right) /\left|\mathbf{r}-\mathbf{r}_{i}\right|$ in $3 \mathrm{D}$; and $V\left(\mathbf{r}-\mathbf{r}_{i}, \xi\right)=K_{0}\left(\left|\mathbf{r}-\mathbf{r}_{i}\right| / \xi\right)$ in $2 \mathrm{D}$, where $K_{0}$ is the zeroth-order modified Bessel function. Evaluating Eq. (37) at the boundary of the droplet $\left(\mid \mathbf{r}-\mathbf{r}_{i}\right.$ $\left.\mid=R_{i}\right)$ gives

$$
\frac{1}{R_{i}}=\chi(t)-Q_{i} V\left(R_{i}, \xi, t\right)
$$

We convert the discrete definition of average of a function $h(R),\langle h(R)\rangle=[1 / N(t)] \Sigma_{i} h\left(R_{i}\right)$, into a continuum definition, $\overline{h(R)}=\int_{0}^{\infty} h(R) f(R, t) d R / \int_{0}^{\infty} f(R, t) d R$. Using Eqs. (14) and (31), we can write

$$
\begin{aligned}
\sum_{i} Q_{i} & =N(t)\left\{\overline{[V(R, \xi, t)]^{-1}} \chi(t)-\overline{[R V(R, \xi, t)]^{-1}}\right\} \\
& =-\frac{1}{a} \frac{\partial \chi}{\partial t}-Q_{n u c l}=\frac{1}{a} \xi^{-2} \chi(t)-\frac{1}{a} S_{b}+\frac{1}{a} S_{n}-Q_{n u c l} .
\end{aligned}
$$

Thus, we can identify $I(R)=a / V(R, \xi, t)$ and

$$
\xi^{-2}=a \int_{0}^{\infty} \frac{f(R, t)}{V(R, \xi, t)} d R,
$$




$$
S_{b}=a \int_{0}^{\infty} \frac{f(R, t)}{R V(R, \xi, t)} d R,
$$

and, $S_{n}=a Q_{n u c l}$ according to Eq. (34). Noting that $Q_{i}$ $=R_{i}^{d-1} \dot{R}_{i}$, and dropping the $i$ subindex, the continuous version of the growth law is, from Eq. (37),

$$
R^{d-1} \dot{R}=\frac{1}{V(R, \xi, t)}\left(\chi(t)-\frac{1}{R}\right),
$$

and $\chi(t)$ is obtained as the time integral of Eq. (31), with Eqs. (34), (41), and (42).

This mean-field approach suffers from the same drawbacks as the original mean-field theory for the Ostwald ripening regime [7]: it is inapplicable to large volume fractions where the screening length $\xi$ is comparable to the average radius of the droplet, since under the Thomas-Fermi approximation, the droplets are treated as point sources and sinks. For example, there is no solution for this set of equations for $\phi>0.085$ in $2 \mathrm{D}$ and for $\phi>0.06$ in $3 \mathrm{D}$.

\section{RESULTS AND DISCUSSION}

Time evolution is determined by three parameters: the nucleation parameter $\chi_{0}$, the width $d R_{c}$ of the nucleation rate distribution function, and the total volume fraction $\phi$. The first two determine the initial radius distribution function and the subsequent crossover behavior, while the effects of $\phi$ persist to late times. The time evolution can be divided into three stages: nucleation, diffusive growth, and coarsening. Nucleation of droplets produces the initial depletion of the supersaturation, which decays faster for low $\chi_{0}$ and high $\phi$. While nucleation is taking place, the first nuclei start to grow, seizing material from the background supersaturation $\chi(t)$. The diffusive growth stage is marked by the high increase in slope of the mean radius $R(t)$ and the corresponding decrease in the slope of the supersaturation $\chi(t)$. During this period, the droplets scarcely interact, and their number stays nearly constant. Finally, when the supersaturation is sufficiently reduced, its role is confined to mediating the exchange of material between individual droplets. The critical radius for nucleation becomes slaved to the mean radius of droplets, i.e., $R_{c}=1 / \chi(t)=R(t)$ [i.e., $C_{0}(t) \rightarrow 0$ ]. Growth is a global, interactive phenomenon, and time evolution proceeds through Ostwald ripening.

Figure 1 shows the droplets and background diffusion field for the time evolution of a $2 \mathrm{D}$ system with volume fraction $\phi=0.05$. In the initial nucleation regime, the background is homogeneous. When the number of droplets $N(t)$ reaches its maximum, the structure in the background signals the imminent decay of $N(t)$. In the diffusive regime, most droplets are growing at the expense of the background supersaturation. Note that they are located in the depleted white or light gray regions. Finally, the ripening regime shows a clearly correlated structure in the background.

Figure 2 shows the dependence of the mean radius $R(t)$, the droplet number per unit volume $n(t)$, and the supersaturation $\chi(t)$ on $\chi_{0}$ and $\phi$ for 3D systems. The top row compares different values of $\chi_{0},(1 / 7,1 / 6,1 / 5$, and 1/4.5) for a volume fraction $\phi=0.05$; and a nucleation rate width proportional to the interface $\left(d R_{c}=0.05 R_{c}\right)$. The bottom row
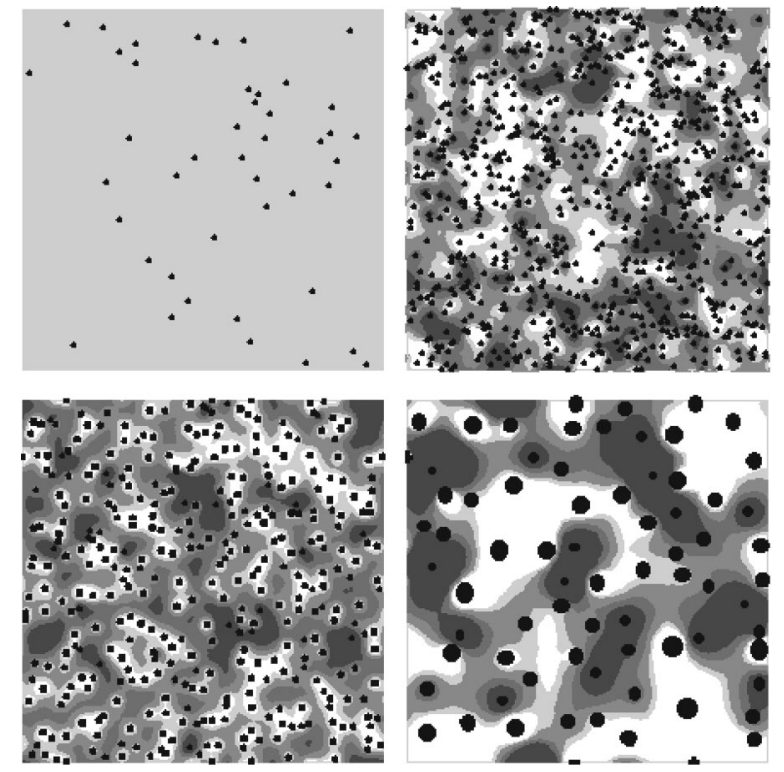

FIG. 1. Time evolution describing nucleation, growth and coarsening for a two-dimensioinal system with volume fraction $\phi$ $=0.05$ and $\chi_{0}=1 / 2$. Lengths have been scaled by the side $L$ of the box, so that the picture has side equal to 1 . Top left: nucleation regime $(t=9500)$. Top right: configuration for the maximum number of droplets $\left(t=1.15 \times 10^{5}\right)$. Bottom left: diffusive growth regime $\left(t=4.79 \times 10^{5}\right)$. Bottom right: ripening regime $(t=5.7$ $\left.\times 10^{7}\right)$. The gray scale used in the background is relative to the supersaturation $\chi(t)$ for the corresponding time. The darkest gray corresponds to $[\theta(\mathbf{r}, t)-\chi(t)] / \chi(t) \geqslant 0.12$; the lightest gray corresponds to $-0.04 \leqslant[\theta(\mathbf{r}, t)-\chi(t)] / \chi(t)<0.04$ and the white color to $[\theta(\mathbf{r}, t)-\chi(t)] / \chi(t) \leqslant-0.08$. Large droplets generally are in the white region, where there is depletion of the supersaturation, while disintegrating droplets are in the dark gray regions of high diffusion fields. To facilitate visualization, droplets are drawn about three times larger their size. Generally, even the nearest droplets are at least two or more radii away.

compares different values of $\phi(0.04,0.065$, and 0.083$)$ for $\chi_{0}=1 / 6$ and a nucleation rate density width $d R_{c}$ $=\left(\sqrt{6} \chi_{0}\right)^{-1}$. As expected, the nucleation parameters $\chi_{0}$ and $d R_{c}$ are irrelevant for the late stages, and the functions $R(t), n(t)$, and $\chi(t)$ collapse onto correspondingly $\phi$-dependent universal functions. This is clearly shown in the top row of Fig. 2, where the three functions $R(t), n(t)$, and $\chi(t)$, evaluated at different values of $\chi_{0}$, collapse onto $\chi_{0}$-independent functions at late stages. The apparent overlapping at late times of the functions $n(t)$ corresponding to different $\phi$ is due to the relative displacement of the maximum of $n(t)$. We discuss the late-stage, $\phi$-dependent universal function for $R(t)$ below.

The crossover to the $\phi$-dependent scaling form can be relatively fast, or take several decades in time, depending on the nucleation history of the system. In the mean-field approach $[15,3,4]$, it has been customary to rescale lengths by $\chi_{0}$ and time by $\chi_{0}^{3}$. The nucleation rate is then expressed in terms of the variable $y=\chi(t) / \chi_{0}$ [with $y_{0}=y(t=0)$ $\left.=\phi / \chi_{0}\right]$. We will discuss this rescaling later. For now we concentrate on the activation energy, which in $d$ dimensions is given by $E_{c} /(k T)=1 / y^{d-1}$. Thus, the nucleation rate increases and the maximum of $n(t)$ is higher and occurs sooner with large $y$, i.e., with decreasing $\chi_{0}$ and increasing 

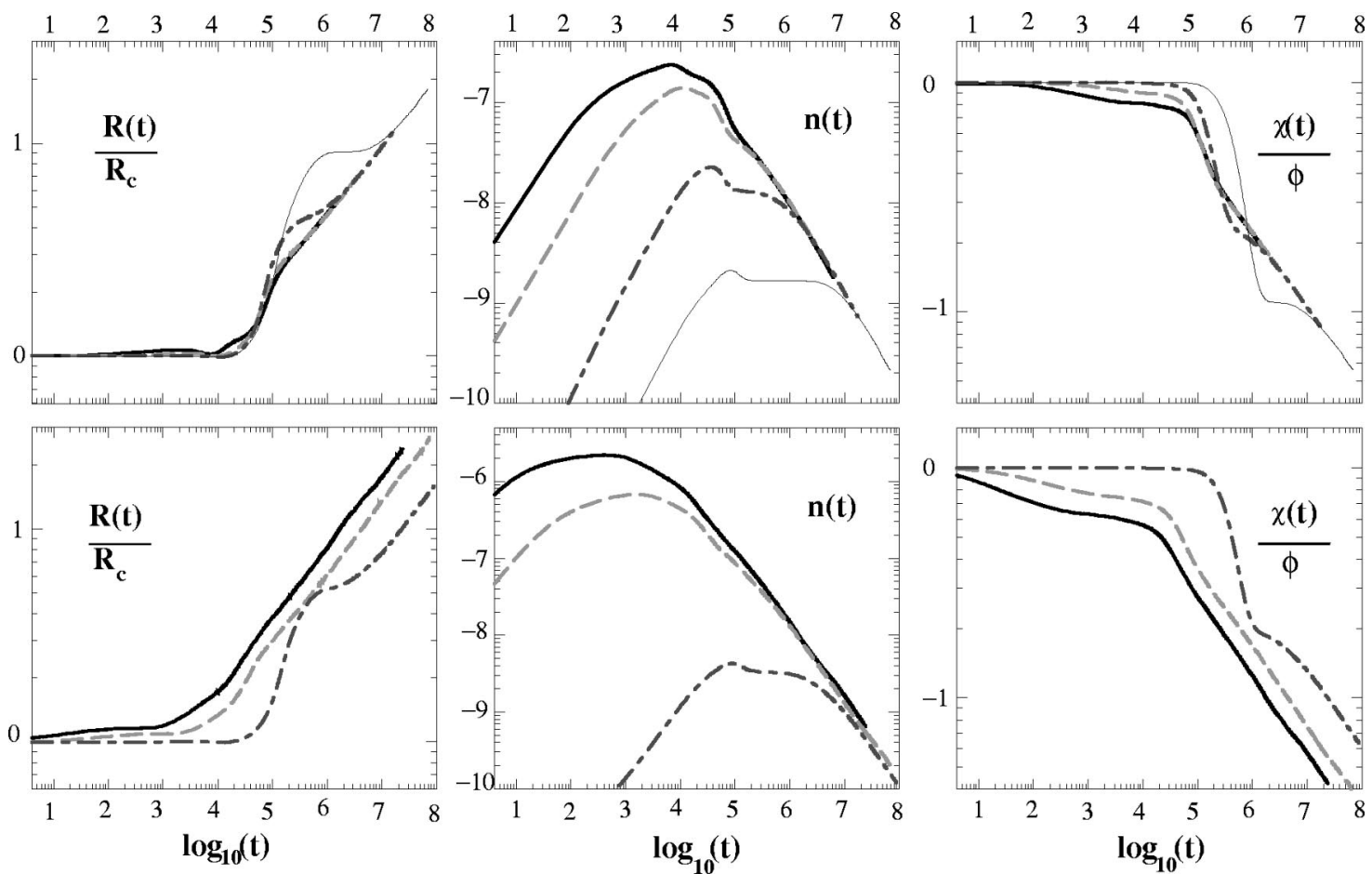

FIG. 2. Left, middle, and right panels show log-log plots for the mean radius $R(t)$ (divided by the critical radius $R_{c}$ ), the droplet number per unit volume $n(t)$, and the supersaturation $\chi(t)$ [divided by the initial supersaturation $\chi(t=0)=\phi$ ] for a three-dimensional system. Top row: $\phi=0.05$ and $\chi_{0}=1 / 7$ (thick solid line), 1/6 (long-dash line), 1/5 (short-dash line), and 1/4.5 (thin solid line). The nucleation rate density width is chosen as $d R_{c}=0.05 R_{c}$. Bottom row: $\chi_{0}=1 / 6$ and $\phi=0.083$ (thick solid line), 0.065 (long-dash line), and 0.04 (short-dash line); $d R_{c}=\left(\sqrt{6} \chi_{0}\right)^{-1}$

$\phi$. For small $\chi_{0}$ and not too large volume fraction, the initial depletion of $\chi(t)$ is rapid and diffusive growth is not important, favoring a relatively early onset of Ostwald ripening, with its characteristic power-law behavior. In agreement with previous results [15], this behavior takes place for $y_{0} \geqslant 0.3$. It is depicted in Fig. 2 for volume fractions $\phi=0.05-0.065$ and $\chi_{0}=1 / 6$ and $1 / 7$.

For $y_{0} \leqslant 0.25$, however, diffusive growth plays an important role. In Fig. 2 this is seen for $\phi=0.05, \chi_{0}=1 / 5\left(y_{0}\right.$ $=0.25) ; \phi=0.04, \chi_{0}=1 / 6\left(y_{0}=0.24\right) ; \quad$ and $\phi=0.05, \chi_{0}$ $=1 / 4.5\left(y_{0}=0.22\right)$. Consider this last case, represented by the thin solid line in the top panel of Fig. 2. At $t \sim 5 \times 10^{4}$, the value of $\chi(t)$ is still large. The few droplets which have nucleated have nearly equal radii. The excess of supersaturation is eliminated by the positive diffusive growth of all droplets, which hardly interact. Thus their number stays constant, signaled by a plateau in the function $n(t)$ and a narrow droplet distribution function, while $R(t)$ markedly increases producing an abrupt decrease in $\chi(t)$. The diffusive growth regime lasts about a decade. For $t>10^{6}, \chi(t)$ has decreased substantially, and is well below what would be its corresponding asymptotic value at the same time. Hence, droplets can no longer grow at its expense. Ostwald ripening then takes over as the dominant mechanism of phase separation.
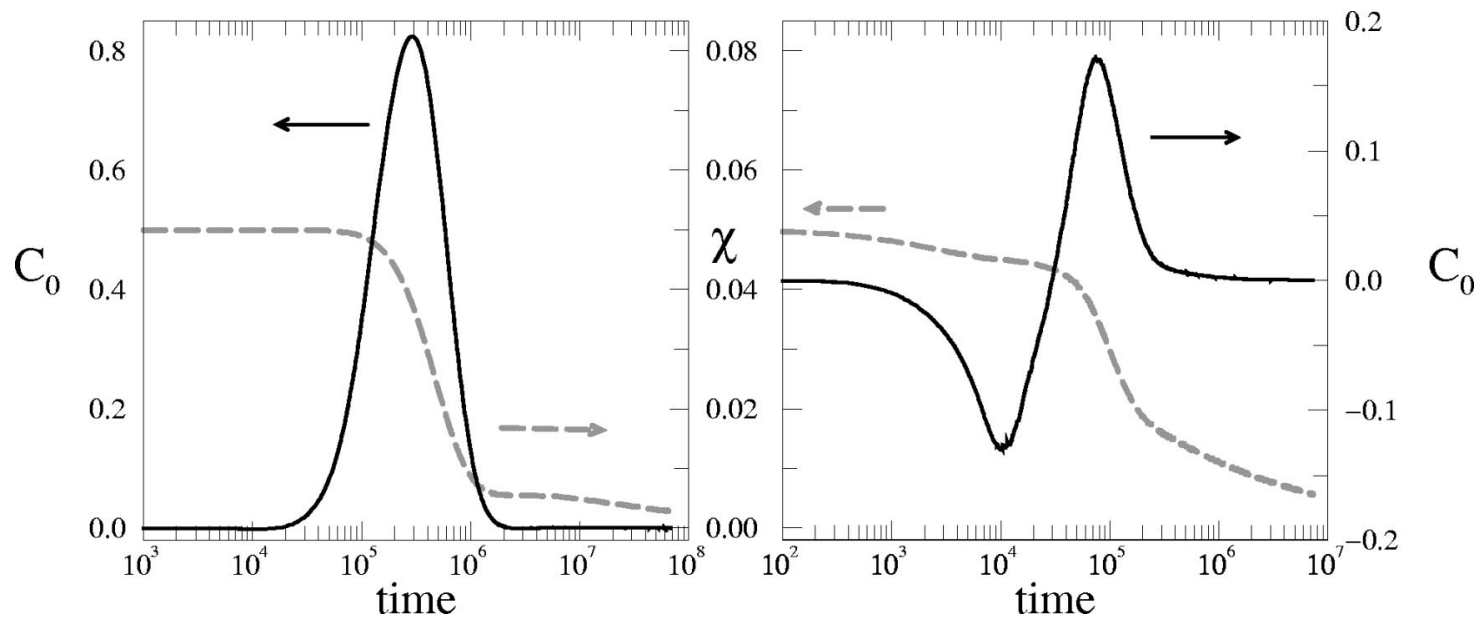

FIG. 3. Logarithmic-linear plot showing the supersaturation $\chi(t)$ (inner axes) and the quantity $C_{0}(t)=Q_{0}(t)-1 / R(t)$ (outer axes) for $\phi=0.05$. The left panel corresponds to $\chi_{0}=1 / 4.5$ and the right panel to $\chi_{0}=1 / 6$. 

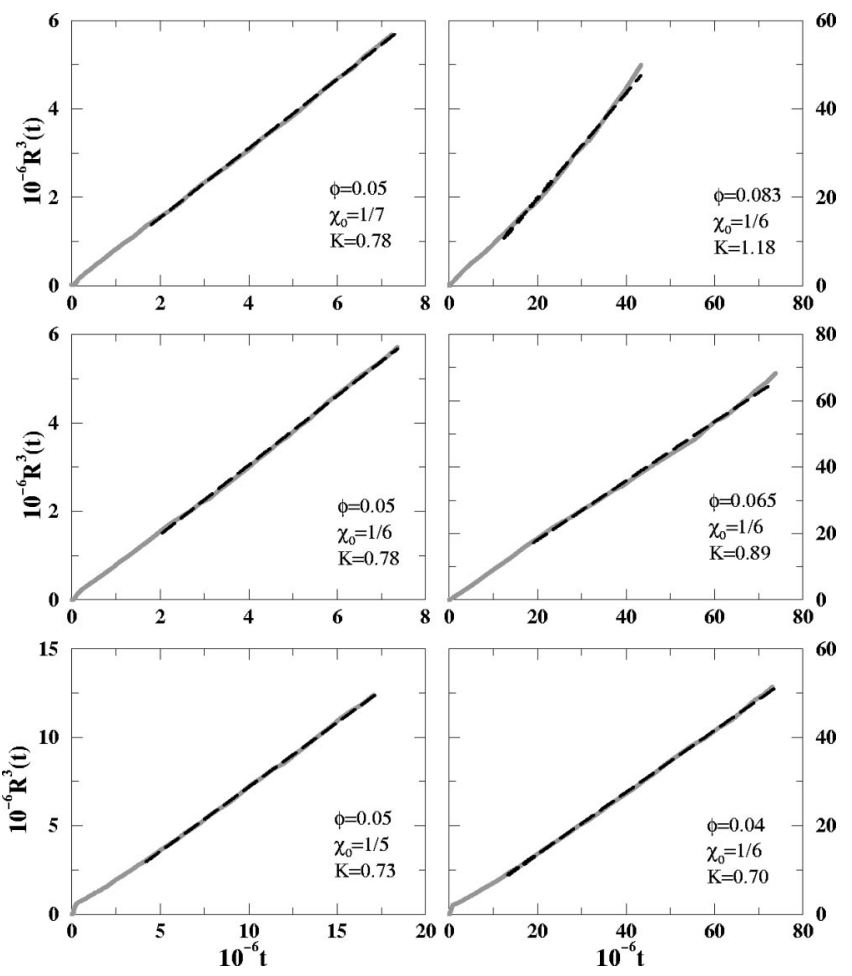

FIG. 4. Plots of $R^{3}(t)$ vs $t$ (gray solid line) and corresponding linear fitting (long-dashed line). The left column corresponds to $\phi$ $=0.05$ and different values of $\chi_{0}$. The three different values of $\chi_{0}$ produce a consistent coarsening coefficient $K(\phi=0.05)$. The right column corresponds to $\chi_{0}=1 / 6$ and different values of $\phi$. The coarsening coefficient $K(\phi)$ increases monotonically with volume fraction.

However, because the droplet distribution function is still narrow, it takes some time for the system to develop a proper dispersion of radii, large enough for the ripening mechanism to become dominant. This "time lag" can be seen in the range $t \sim 10^{6}-10^{7}$, where there are plateaus in $R(t)$ and $\chi(t)$, while $n(t)$ decreases, as droplets slowly disappear.

Figure 3 illustrates the behavior of the coefficient $C_{0}(t)$ $=Q_{0}(t)-1 / R(t)$, where $Q_{0}(t)$ was introduced in Eq. (16) above, for the system just described. This coefficient is important during the diffusive regime. It is near zero during nucleation, but then exhibits large variation during the diffu- sive growth regime, when the supersaturation falls abruptly. It again becomes zero as the system reaches the steady-state Ostwald ripening regime, where the supersaturation becomes slaved to the mean radius, $Q_{0}(t) \simeq \chi(t) \simeq 1 / R(t) \simeq t^{-1 / 3}$.

Figure 4 shows linear fittings for $R^{3}(t)$, for different parameters. The left column corresponds to $\phi=0.05$, and different values of $\chi_{0}$. These plots confirm that different values of $\chi_{0}$ give, for late stages, a consistent coarsening rate $K(\phi)$ independent of $\chi_{0}$. The right column corresponds to $\chi_{0}$ $=1 / 6$, and different values of $\phi$. These plots also confirm that the coarsening rate $K(\phi)$ is a monotonically increasing function of the volume fraction $\phi$. The value for $\phi=0.083$ is only indicative, since this run has not completely reached the scaling regime and there is considerable diffusive behavior still present. For late stages, the dependence of the droplet number density on the coarsening rate $K(\phi)$ is given by $n(t)=n\left(t_{1}\right) R^{d}\left(t_{1}\right) /\left[R^{3}\left(t_{1}\right)+K(\phi) t\right]^{d / 3}[7]$, where $t_{1}$ designates the time at which the linear fitting starts in each plot, and $R\left(t_{1}\right)$ is the corresponding value of the mean radius. The linear fittings of $n\left(t_{1}\right) R^{3}\left(t_{1}\right) / n(t)$ vs. $t$ produce the same coarsening rate $K(\phi)$ as the one obtained with $R^{3}(t)$ and shown in Fig. 4.

Figure 5 shows the dependence of the mean radius $R(t)$, the droplet number per unit volume $n(t)$, and the supersaturation $\chi(t)$ on $\chi_{0}$ and $\phi$ for two-dimensional systems. Three values of $\chi_{0}(1 / 4,1 / 3$, and $1 / 2)$, are shown for a volume fraction $\phi=0.05$. The thin solid line, on the other hand, shows the corresponding quantities for $\phi=0.10$ and $\chi_{0}$ $=1 / 3$. For these plots, the nucleation rate width is taken as proportional to the interface $\left(d R_{c}=0.05 R_{c}\right)$. The dependence of these functions upon $\phi$ and $\chi_{0}$ agrees with the three-dimensional case. The nucleation parameters $\chi_{0}$ and $d R_{c}$ are irrelevant for the late stages, and the functions $R(t), n(t), \quad$ and $\quad \chi(t)$ collapse onto correspondingly $\phi$-dependent universal functions. For $\phi=0.05$, the coarsening coefficient is $K(0.05) \sim 0.43$ for $\chi_{0}=1 / 4$ and $1 / 3 ; \chi_{0}$ $=1 / 2$ still has not reached this value, giving $K(0.05) \sim 0.39$ for the last stage reached by the simulation. The run with $\phi=0.10$ has not completely reached the scaling regime. For the last stages shown in the figure, there is a measured coarsening coefficient $K(0.10) \sim 0.94$, although we expect this value to change for later times. The late-stage, $\phi$-dependent results obtained both in $2 \mathrm{D}$ and $3 \mathrm{D}$ are consistent with pre-
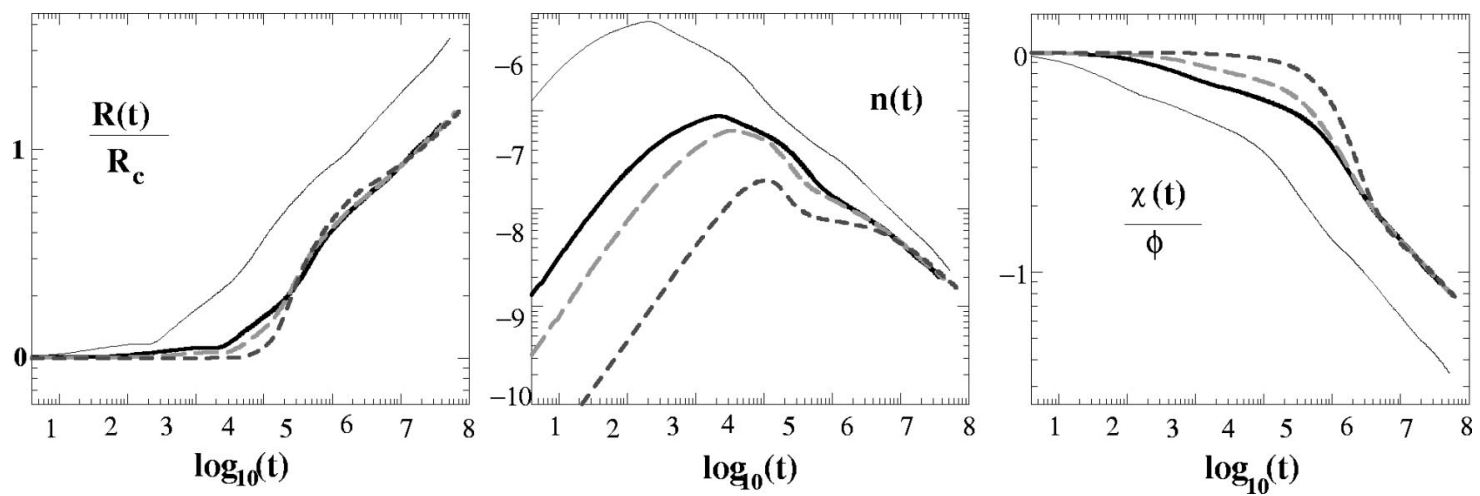

FIG. 5. Left, middle, and right panels show $\log$-log plots for the mean radius $R(t) / R_{c}$, the droplet number per unit volume $n(t)$, and the supersaturation $\chi(t) / \phi$ for a two-dimensional system. The thin solid line corresponds to $\phi=0.10$ and $\chi_{0}=1 / 3$. The other three lines correspond to $\phi=0.05$ and different values of $\chi_{0}: \chi_{0}=1 / 4$ (thick solid line), 1/3 (long-dashed line), and 1/2 (short-dashed line). The nucleation rate density width is chosen as $d R_{c}=0.05 R_{c}$ for all cases. 
vious results for late stages [6-8] and are a consequence of having incorporated the correlations among droplets in the dynamical equations.

Figure 6 shows the completion time $\tau$, defined by $\chi(t$ $=\tau)=\chi(t=0) / 2=\phi / 2$ for both $2 \mathrm{D}$ and 3D. As has been pointed out [24], the completion time is an experimentally convenient quantity for studying nucleation. To estimate a completion time, one must consider both droplet formation and growth. The third panels in Figs. 2 and 5 clearly show that $t=\tau$ occurs during the growth stage. Figure 7 shows two schematic diagrams of the different regimes, nucleation, diffusive growth and ripening, and their corresponding crossover regions, as function of the nucleated volume $\chi_{\nu}=1$ $-\chi(t) / \phi$ and $\phi$ (left panel) or $\chi_{0}^{-1}$ (right panel).

Some initial and intermediate times for the radius distribution functions $f(R, t)$ for $\phi=0.05$ and $\chi_{0}=1 / 6$ are shown in Fig. 8. The top panels show $R(t)$, the droplet number per unit volume $n(t)$ and the critical radius $R_{c}(t)=1 / \chi(t)$. The vertical bars indicate the time at which the droplet distribution functions are depicted in the lower panels. The three columns differ in the width $d R_{c}$. The left column corresponds to $d R_{c}=0$, the middle column to $d R_{c}=0.05 R_{c}$, and the right column to $d R_{c}=\left(\sqrt{6} \chi_{0}\right)^{-1}$. The solid line represents the results of the mean-field theory that includes the droplet correlation effects. The left column corresponds to $d R_{c}=0$, i.e., when all the droplets are nucleated with the critical radius. This extreme situation depicts clearly the different mechanisms of nucleation, growth, and ripening.

With the nucleation of critical droplets, $f(R, t)$ develops a high peak centered at $R_{c}$. As supersaturation diminishes, $R_{c}$ increases and the newly nucleated droplets have larger radii than the older ones, so that $f(R, t)$ is asymmetric, as shown in the light-gray shaded distribution $f\left(R, t=t_{1}\right)$. The "low shoulder" of the distribution at $R \simeq 18$ corresponds to the first nucleated droplets, while the high peak at $R \simeq 22$ corresponds to the newer droplets. The maximum number of droplets is reached at $t=t_{2}$. Before this time none of the originally nucleated droplets have disappeared. The abrupt dissolution of many subcritical droplets after $t_{2}$ leads to a sharp decay in $n(t)$, for $t>t_{2}$. The distribution function $f\left(R, t=t_{2}\right)$ has a high peak for $R \simeq 22>\langle R(t)\rangle$. At the right

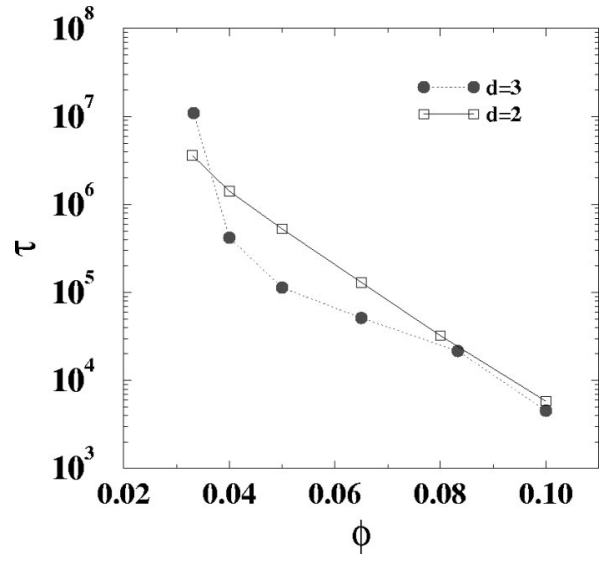

FIG. 6. Completion time $\tau$ as function of the volume fraction $\phi$, for two-dimensional systems (empty squares) and threedimensional systems (filled circles). The nucleation rate density width is $d R_{c}=\left(0.5 R_{c} / \chi_{0}\right)^{1 / 2}$ for $2 \mathrm{D}$ and $d R_{c}=\left(\sqrt{6} \chi_{0}\right)^{-1}$ for $3 \mathrm{D}$.

of this peak, the distribution falls very rapidly to zero, and at the left of the peak, the distribution has a long tail. This excessive population of small droplets causes $\langle R(t)\rangle$ to decrease towards the kink at $t_{2}$. Immediately after $t_{2}$, these small droplets dissolve, $n(t)$ decreases and $\langle R(t)\rangle$ increases sharply while $f(R, t)$ becomes almost symmetric with a small tail for $R<\langle R(t)\rangle$. The dissolution of the small droplets allows a $\chi(t)$ high enough to nucleate new droplets. Intensive nucleation and dissolution of small droplets occurs between $t_{2}$ and $t_{4}$ and the new droplets produce the second kink of $n(t)$ at $t_{4}$. The coarsening of droplets produces a hump in $f\left(R, t=t_{4}\right)$ for $R>\langle R(t)\rangle$, which becomes a second peak between $t_{4}$ and $t_{5}$, creating a bimodal distribution $f(R, t)$ and a second kink in $\langle R(t)\rangle$. At exactly this point, $R_{c}(t)$ crosses $\langle R(t)\rangle$, ending a subcritical stage, with many droplets smaller than the critical size. After $t_{5}$ the peak for $R<\langle R(t)\rangle$ rapidly decreases, while the coarsening peak increases and moves towards larger $R$ 's.

The middle column in Fig. 8 shows the case when the width $d R_{c}$ is comparable to the interface thickness. The mechanisms are similar but "washed out" due to the dispersion in radii, and the subcritical stage ends sooner. These

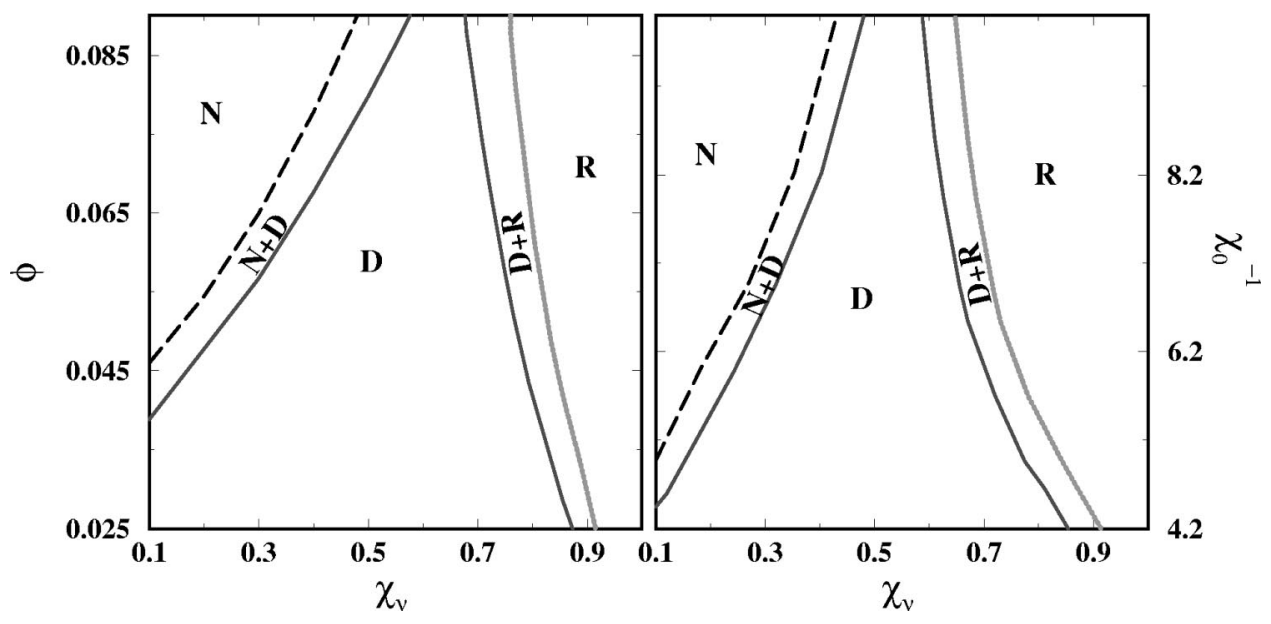

FIG. 7. Schematic diagram of the different regimes, nucleation (N), diffusion (D), and ripening (R) as function of $\phi$ (left) or $\chi_{0}^{-1}$ (right) and the nucleated volume $\chi_{\nu}=1-\chi(t) / \phi$. The narrow regions between $\mathbf{N}$ and $\mathbf{D}$ and between $\mathbf{D}$ and $\mathbf{R}$ indicate a crossover between the corresponding regimes. The left panel, $\phi-\chi_{\nu}$, is obtained for $\chi_{0}=1 / 6$ and the right panel, $\chi_{0}^{-1}-\chi_{\nu}$, is obtained for $\phi=0.05$. 

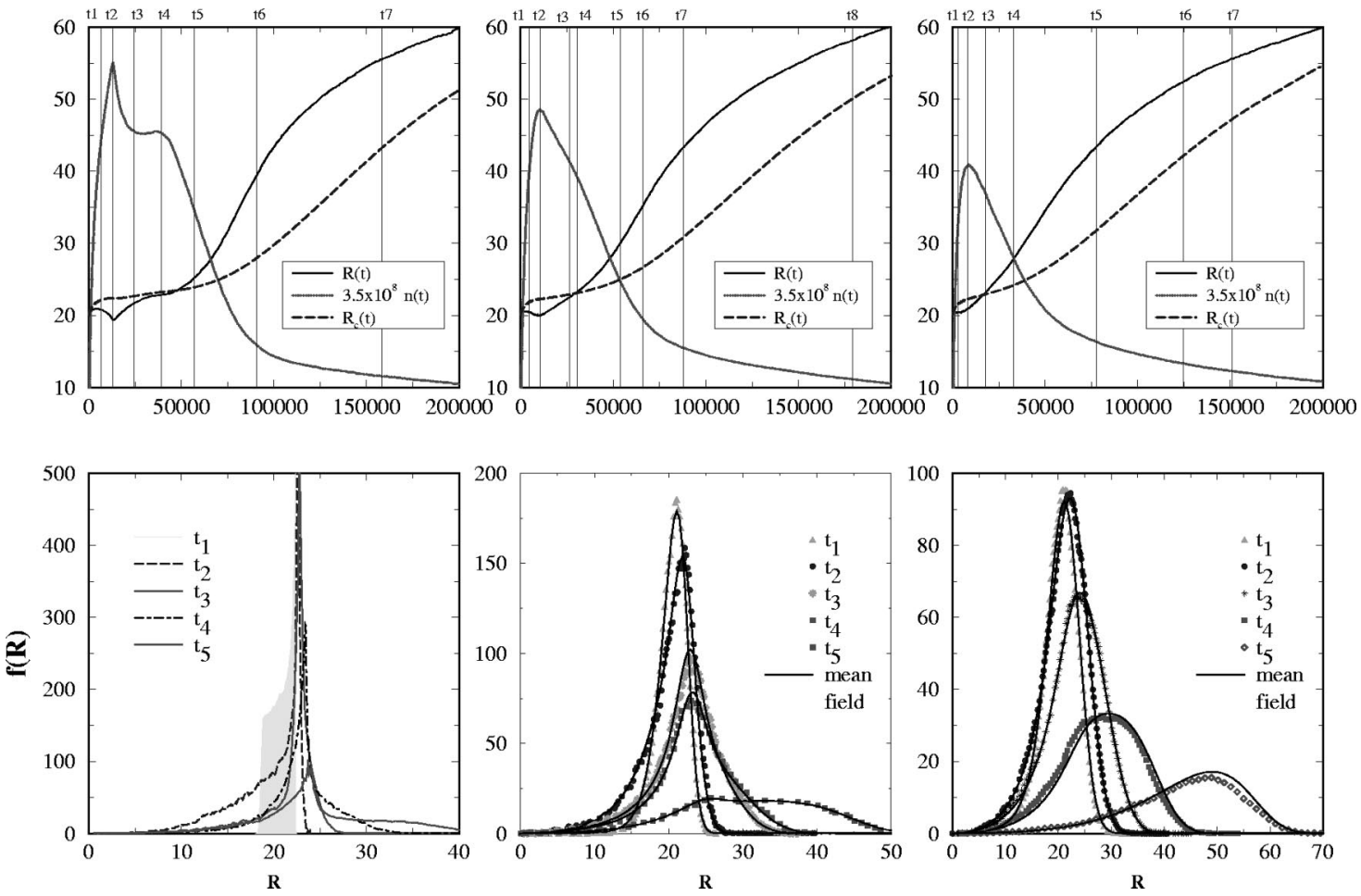

FIG. 8. These plots correspond to a three-dimensional system with $\phi=0.05$ and $\chi_{0}=1 / 6$. The top panels show the mean radius $R(t)$, the droplet number per unit volume $n(t)$, and the critical radius $R_{c}(t)=1 / \chi(t)$. The vertical bars indicate the time at which the droplet distribution functions are depicted in the lower panels. The three columns differ in the width $d R_{c}$. The left column corresponds to $d R_{c}$ $=0$, the middle column to $d R_{c}=0.05 R_{c}$, and the right column to $d R_{c}=\left(\sqrt{6} \chi_{0}\right)^{-1}$. Each of these functions is obtained by averaging 25 runs with a maximum number of nucleated droplets $\simeq 1000$. The solid line represents the results of the mean-field theory that includes the droplet correlation effects.
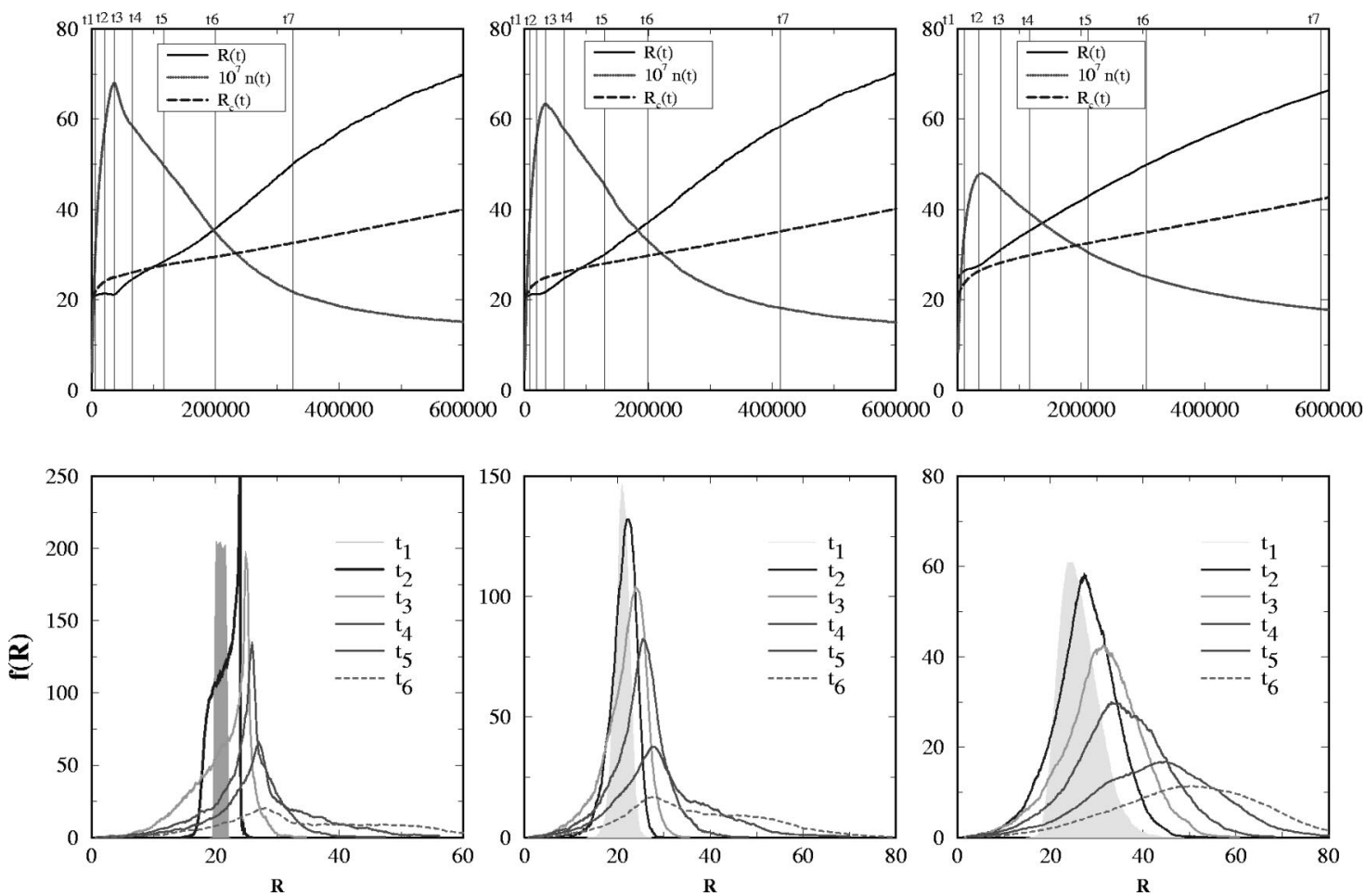

FIG. 9. These plots correspond to a two-dimensional system with $\phi=0.05$ and $\chi_{0}=1 / 3$. The top panels show the mean radius $R(t)$, the droplet number per unit volume $n(t)$, and the critical radius $R_{c}(t)=1 / \chi(t)$. The vertical bars indicate the time at which the droplet distribution functions are depicted in the lower panels. The three columns differ in the width $d R_{c}$. The left column corresponds to $d R_{c}$ $=0$, the middle column to $d R_{c}=0.05 R_{c}$, and the right column to $d R_{c}=\left(0.5 R_{c} / \chi_{0}\right)^{1 / 2}$. 

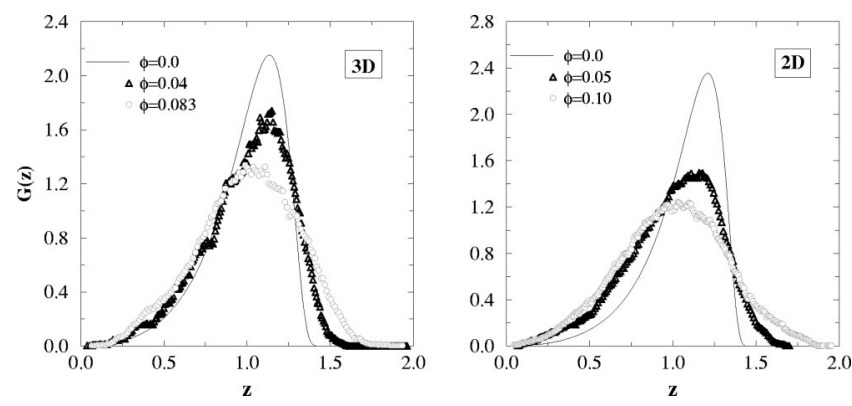

FIG. 10. Scaled droplet distribution functions for threedimensioinal and two-dimensional systems. The thin solid line corresponds to the infinite dilution limit $(\phi=0)$. The finite volume fraction functions shown with the triangles and circles correspond to the late stage coarsening regime.

"smoothening" effects are even greater for the standard width $d R_{c}=\left(\sqrt{6} \chi_{0}\right)^{-1}$, shown in the third column. The solid line distribution functions in the second and third panels represent our mean-field solution to the equations, based on the Thomas-Fermi approximation. These are the best results; the predictions of the mean-field solution tend to worsen with larger $\chi_{0}$ and/or with larger $\phi$.

The same qualitative trends for the distribution function are observed in two-dimensional systems, as shown in Fig. 9. These plots correspond to two-dimensional systems with $\phi$ $=0.05$ and $\chi_{0}=1 / 3$. As before, the top panels show the mean radius $R(t)$, the droplet number per unit volume $n(t)$, and the critical radius $R_{c}(t)=1 / \chi(t)$, while the vertical bars indicate the time at which the droplet distribution functions are depicted in the lower panels. The three columns differ in the width $d R_{c}$. The left column corresponds to critical droplets $\left(d R_{c} \simeq 0\right)$, the middle column to droplets nucleated with a width proportional to the interface $\left(d R_{c}=0.05 R_{c}\right)$, and the right column to the standard width $d R_{c}=\left(0.5 R_{c} / \chi_{0}\right)^{1 / 2}$.

Figure 10 shows the scaled droplet distribution functions for three-dimensional and two-dimensional systems. The thin solid line corresponds to the infinite dilution limit $(\phi=0)$, and is obtained through an exact analytical expression given by Lifshitz and Slyozov [5]. The finite volume fraction functions shown with the triangles and circles correspond to the late stage coarsening regime: $\phi=0.04$ (triangle) and $\phi$ $=0.083$ (circle) for the three-dimensional system, and $\phi$ $=0.05$ (triangle) and $\phi=0.10$ (circle) for the twodimensional system. The effects of nonzero volume fraction is to lower the peak and widen the width of the distribution function, which becomes more symmetric for higher volume fraction. The dependence on the volume fraction of both the distribution function and the coarsening rate are features that arise naturally in our formalism, and that are absent in the Langer-Schwartz model.

Figure 11 shows a comparison between the simulation results, our Thomas-Fermi analytical solution and the integration of the isolated-droplet, mean-field continuity equation. The figure shows the mean droplet radius $R(t)$ and the droplet density $n(t)$ for a three-dimensional system with $\phi$ $=0.05, \chi_{0}=1 / 6$, and $d R_{c}=0.05 R_{c}$. The Thomas-Fermi approximation (long-dashed line) agrees very well with the simulation results (thick black line). The agreement is good for small $\chi_{0}$, and conversely, deteriorates for larger $\chi_{0}$ (or larger $\phi$ ). The integration of the continuity equation (short-
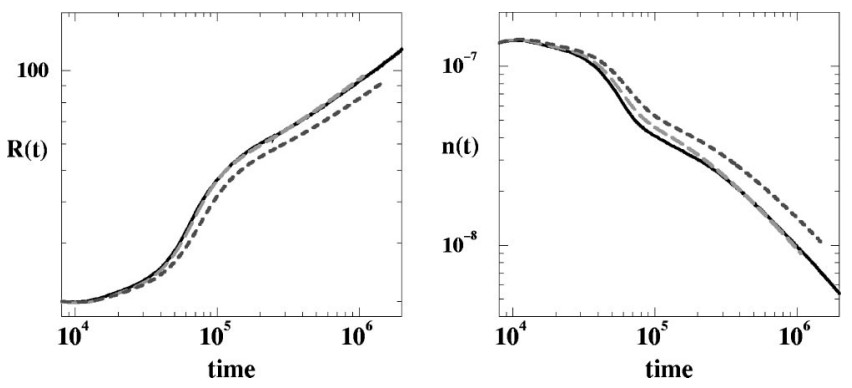

FIG. 11. For a three-dimensional system with $\phi=0.05, \chi_{0}$ $=1 / 6$, and $d R_{c}=0.05 R_{c}$, we compare the results of our simulation (thick black line), the Thomas-Fermi approximation (long-dashed line) and the integration of the continuity equation (short-dashed line).

dashed line) has been performed straightforwardly, without the various assumptions used by Langer-Schwartz. We have also eliminated the restriction of nucleating only supercritical droplets. Naturally, the agreement with the simulation results deteriorates for later times.

Concerning scaling, we note that the original mean-field theory [15] contains one independent variable, $y=\chi(t) / \chi_{0}$. Therefore, all the results can be expressed in terms of this variable and the nucleation rate width, if one defines new radii $\rho$ and time $\tau$ as $\rho=\chi_{0} R$ and $\tau=\chi_{0}^{3} t$. However, this requires the coarsening rate $R^{3}(t) / t=\rho^{3}(\tau) / \tau$ to be independent of $\phi$, which it is not. Figure 12 compares the results for $y_{0}=0.32$, considering $\left(\phi, \chi_{0}\right)=(0.08,1 / 4)$ and $(0.04,1 / 8)$; and the mean-field integration for $y_{0}=0.32$. Notice that it is only possible to collapse the curves for different volume fractions during the initial and early times.

In conclusion, we have introduced a new model for nucleation and growth that combines steady-state homogeneous nucleation theory with the Ostwald ripening mechanism and includes the correlations among droplets. These correlations originate in the interaction of the diffusional fields corre-

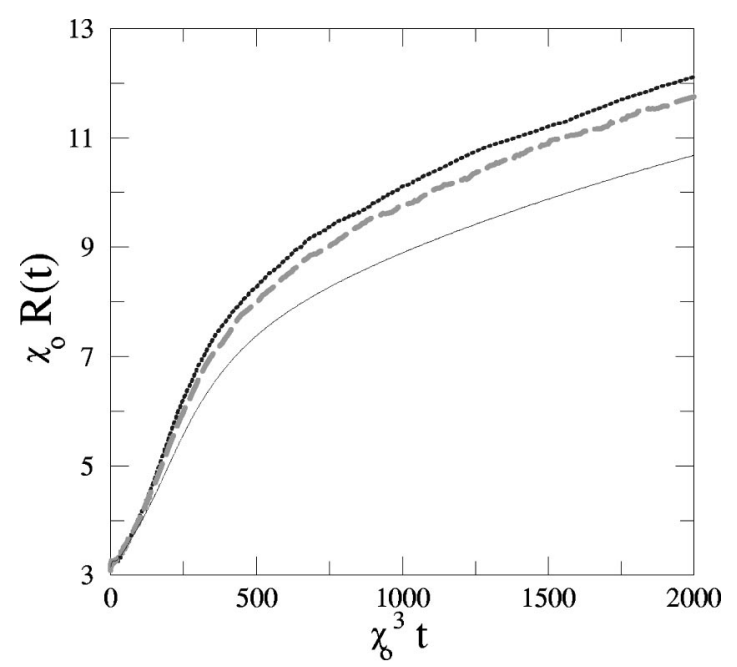

FIG. 12. Comparison of the results obtained for $y_{0}=0.32$ as obtained for $\phi=0.08, \chi_{0}=1 / 4$ (dark dotted line); $\phi=0.04, \chi_{0}=1 / 8$ (gray dashed line); and the $\phi=0$ mean-field integration for $y_{0}$ $=0.32$ (thin solid line). The scaled mean radii, $R(t) \chi_{0}$, as function of the scaled time, $t \chi_{0}^{3}$, agree during the initial and early times, but as soon as the droplets start to interact, the curves start to depart. 
sponding to each precipitate, and thus depend on the relative position of the droplets. The set of self-consistent equations naturally incorporates the crossover from the early-stage nucleation to the late-stage scaling regime. We have presented simulations on both two and three dimensions. We have also presented a mean-field, Thomas-Fermi approximation that includes the effect of these correlations. A good experimental candidate to test our results would be a latticematched binary alloy during solid-state precipitation.

\section{ACKNOWLEDGMENTS}

We thank Dean Stinson-O'Gorman for his collaboration in the earlier stages of this work. This work was supported by the Natural Sciences and Engineering Research Council of Canada, and le Fonds pour la Formation de Chercheurs et l'Aide à la Recherche du Québec.

\section{APPENDIX}

The multidroplet diffusion equation has a retarded Green's function associated with it, which can be written in $d$ dimensions as

$$
\begin{aligned}
G_{d}\left(\mathbf{r}, \mathbf{r}^{\prime}, t, t^{\prime}\right) & =\frac{a}{\left[4 \pi\left(t-t^{\prime}\right)\right]^{d / 2}} \exp \left(-\frac{\left|\mathbf{r}-\mathbf{r}^{\prime}\right|^{2}}{4\left(t-t^{\prime}\right)}\right) \\
& =\frac{a}{(2 \pi)^{d}} \int d^{d} k e^{-k^{2}\left(t-t^{\prime}\right)} e^{i \mathbf{k} \cdot\left(\mathbf{r}-\mathbf{r}^{\prime}\right)}
\end{aligned}
$$

where, as before, $a=2 \pi$ in $2 \mathrm{D}$ and $a=4 \pi$ in 3D. A formal solution of Eq. (10) in terms of the Green's function is

$$
\theta(\mathbf{r}, t)=\chi(t)-a \sum_{j=1}^{N} \int_{0}^{t} d s \exp \left(-\frac{\left|\mathbf{r}-\mathbf{r}_{j}\right|^{2}}{4 s}\right) \frac{Q_{j}(t-s)}{(4 \pi s)^{d / 2}}
$$

The steady-state solution of the previous equation is obtained by taking the limit $t \rightarrow \infty$ in the second term. This is more easily done by using the Fourier transform of $G_{d}\left(\mathbf{r}-\mathbf{r}^{\prime}, t\right.$ $\left.-t^{\prime}\right)$ :

$$
\begin{gathered}
\lim _{t \rightarrow \infty} a \sum_{j=1}^{N} \int_{0}^{t} d s Q_{j}(t-s) \frac{\exp \left[-\frac{\left|\mathbf{r}-\mathbf{r}_{j}\right|^{2}}{4 s}\right]}{(4 \pi s)^{d / 2}} \\
=a \sum_{j=1}^{N} Q_{j} \int \frac{d^{d} k}{(2 \pi)^{d}} e^{i \mathbf{k} \cdot\left(\mathbf{r}-\mathbf{r}_{j}\right)} \int_{0}^{\infty} d s e^{-k^{2} s} .
\end{gathered}
$$

In the second term, we recognize the stationary Green's function:

$$
G_{d}\left(\mathbf{r}-\mathbf{r}^{\prime}\right)=a \int \frac{d^{d} k}{(2 \pi)^{d}} \frac{e^{i \mathbf{k} \cdot\left(\mathbf{r}-\mathbf{r}^{\prime}\right)}}{k^{2}}
$$

which can be written as $G_{3}\left(\mathbf{r}-\mathbf{r}^{\prime}\right)=\left|\mathbf{r}-\mathbf{r}^{\prime}\right|^{-1}$ or $G_{2}(\mathbf{r}$ $\left.-\mathbf{r}^{\prime}\right) \simeq \ln \left(\left|\mathbf{r}-\mathbf{r}^{\prime}\right| / L\right)$, where $L$ is the system size. One can solve for the source coefficient $Q_{i}$ by averaging the concentration field over the surface of the $i$ th particle and setting that equal to the local concentration $\theta\left(R_{i}\right)=1 / R_{i}$ :

$$
\begin{aligned}
\frac{1}{R_{i}}= & \chi(t)-Q_{i} G_{d}\left(R_{i}\right)-a \sum_{j \neq i}^{N} \\
& \times \int_{0}^{t} d s \exp \left(-\frac{\left|\mathbf{r}_{i}-\mathbf{r}_{j}\right|^{2}}{4 s}\right) \frac{Q_{j}(t-s)}{(4 \pi s)^{d / 2}} .
\end{aligned}
$$

In this equation, one approximation has been used: the timedependent Green's function in the integral over droplet $i$ has been replaced by the stationary Green's function.
[1] M. Volmer and A. Weber, Z. Phys. Chem. Abt. A 119, 277 (1926); L. Farkas, ibid. 125, 236 (1927); R. Becker and W. Döring, Ann. Phys. (Leipzig) 24, 719 (1935); R. Kaischew and I. N. Stranski, Z. Phys. Chem. Abt. B 26, 81 (1935); A. N. Kolmogorov, Bull. Acad. Sci. USSR, Mat. Ser. 1, 335 (1937); W. A. Johnson and R. F. Mehl, Trans. Am. Inst. Min. Metall. Pet. Eng. 135, 416 (1939); M. Avrami, J. Chem. Phys. 7, 1103 (1939); 8, 212 (1940); 9, 177 (1941); J. B. Zeldovich, Acta Physicochim. URSS 18, 1 (1943).

[2] J. S. Langer, Ann. Phys. (N.Y.) , 41, 108 (1967); 54, 258 (1969); S. Coleman, Phys. Rev. D 15, 2929 (1977).

[3] J. S. Huang, S. Vernon, and N. C. Wong, Phys. Rev. Lett. 33, 140 (1974); J. S. Huang, W. I. Goldburg, and M. R. Moldover, ibid. 34, 639 (1975); N. C. Wong and C. M. Knobler, J. Chem. Phys. 69, 725 (1978); A. J. Schwartz, S. Krishnamurthy, and W. I. Goldburg, Phys. Rev. A 21, 1331 (1980); G. C. Howland, N. C. Wong, and C. M. Knobler, J. Chem. Phys. 73, 522 (1980); S. Krishnamurthy and W. I. Goldburg, Phys. Rev. A 21, 2147 (1980); E. D. Siebert and C. M. Knobler, Phys. Rev. Lett. 52, 1133 (1984); 54, 819 (1985); K. Y. Min and W. I.
Goldburg, ibid. 70, 469 (1993).

[4] D. W. Herrmann, W. Klein, and D. Stauffer, Phys. Rev. Lett. 49, 1262 (1982); D. Stauffer, A. Coniglio, and D. W. Herrmann, ibid. 49, 1299 (1982).

[5] I. M. Lifshitz and V. V. Slyozov, J. Phys. Chem. Solids 19, 35 (1961); C. Wagner, Z. Elektrochem. 65, 581 (1961).

[6] J. A. Marqusee and J. Ross, J. Chem. Phys. 80, 536 (1984); P. W. Voorhees and M. E. Glicksman, Acta Metall. 32, 2001 (1984); J. A. Marqusee, J. Chem. Phys. 81, 976 (1984); P. W. Voorhees, J. Stat. Phys. 38, 231 (1985); C. W. J. Beenakker, Phys. Rev. A 33, 4482 (1986); Y. Enomoto, M. Tokuyama, and K. Kawasaki, Acta Metall. 34, 2119 (1986); M. Marder, Phys. Rev. A 36, 858 (1987); Q. Zheng and J. D. Gunton, ibid. 39, 4848 (1989); A. J. Ardell, Phys. Rev. B 41, 2554 (1990), and references therein; R. Toral, A. Chakrabarti, and J. D. Gunton, Phys. Rev. A 45, 2147 (1992).

[7] J. H. Yao, K. R. Elder, H. Guo, and M. Grant, Phys. Rev. B 45, R8173 (1992); 47, 14110 (1993).

[8] N. Akaiwa and P. W. Voorhees, Phys. Rev. E 49, 3860 (1994); N. Akaiwa and D. I. Meiron, ibid. 54, R13 (1996). 
[9] P. G. Hill, J. Fluid Mech. 25, 593 (1966).

[10] D. A. Porter and K. E. Easterling, Phase Transformations in Metals and Alloys (Chapman and Hall, London, 1992).

[11] S. Q. Xiao and P. Haasen, Acta Metall. Mater. 39, 651 (1991).

[12] H. Yinnon and D. R. Uhlmann, J. Non-Cryst. Solids 54, 253 (1983); V. A. Shneidman, Phys. Rev. Lett. 75, 4634 (1995); J. Chem. Phys. 103, 9772 (1995); A. B. Pevtsov, V. Y. Davydov, N. A. Feoktistov, and V. G. Karpov, Phys. Rev. B 52, 955 (1995)

[13] V. A. Shneidman and P. Hänggi, Phys. Rev. E 49, 641 (1994).

[14] M. Zinke-Allmang, L. C. Feldman, and M. H. Grabow, Surf. Sci. Rep. 16, 377 (1992). and references therein; G. R. Carlow and M. Zinke-Allmang, Phys. Rev. Lett. 78, 4601 (1997).

[15] J. S. Langer and A. J. Schwartz, Phys. Rev. A 21, 948 (1980).

[16] C. Lane Rohrer, M. D. Asta, S. M. Foiles, and R. W. Hyland, Jr., Mathematics of Microstructure Evolution, edited by L. Q. Chen (TMS-Miner. Metals and Mater. Soc., Warrendale, PA, 1996), Vol. 269.

[17] See, for instance, B. B. Rath, Mater. Sci. Eng., B 32, 101 (1995).

[18] P. W. Voorhees and R. J. Schaefer, Acta Metall. 35, 327
(1987); S. C. Hardy and P. W. Voorhees, Metall. Trans. A 19, 2713 (1988).

[19] M. Tokuyama and Y. Enomoto, Phys. Rev. Lett. 69, 312 (1992). See also the experiment by A. Cumming, P. Wiltius, and F. Bates, ibid. 65, 863 (1990). In these studies of droplet growth following heterogeneous nucleation (in contrast to the present study where growth following homogeneous nucleation was investigated), a clear $t^{1 / 2}$ growth law was observed, corresponding to a diffusive growth regime.

[20] Some of this work has been reported previously in the short paper: C. Sagui, D. Stinson O'Gorman, and M. Grant, Phys. Rev. E 56, R21 (1997).

[21] N. J. Günther, D. A. Nicole, and D. J. Wallace, J. Phys. A 13, 1755 (1980).

[22] C. Sagui and R. C. Desai, Phys. Rev. Lett. 74, 1119 (1995).

[23] During growth, some particle overlap occurs. However, for the volume fractions considered here, the fraction of overlapping particles is very small [8]. In the simulations, we implemented a periodic check allowing for coalescence when particles come into contact.

[24] K. Binder and D. Stauffer, Adv. Phys. 25, 343 (1986). 